\title{
Morfometría del Acromion y sus Implicancias Clínicas
}

\author{
Morphometry of Acromion and its Clinical Implications
}

\begin{abstract}
"Alessandra Cavalcanti Torres; **Alice Sá Carneiro Ribeiro; **Danielle Augusta de Sá Xerita Maux; "*Deivson Cavalcante Gomes de Oliveira; ** Gabriela de Moura Neves; ** Raphaella Fernanda Almeida Salgado; * Rodrigo Fragoso de Andrade; "Vitor Caiaffo Brito \& ${ }^{* * *}$ Silvia Regina Arruda de Moraes.
\end{abstract}

TORRES, C. A.; RIBEIRO, C. A. S.; MAUX, S. X. D. A.; OLIVEIRA, G. C. D.; NEVES, M. G.; SALGADO, A. R. F.; ANDRADE, F. R.; BRITO, C. V. \& MORAES, A. S. R. Morfometría del acromion y sus implicancias clínicas. Int. J. Morphol., 25(1):51-54, 2007.

RESUMEN: Estudios previos demostraron que la morfología del acromión y sus respectivas relaciones con el tubérculo supraglenoideo y el proceso coracoides, son de fundamental importancia en la determinación del espacio subacromial. Las variaciones de estas estructuras pueden provocar el principio del síndrome del impacto, también conocido como síndrome del pinzamiento del espacio subacromial. Sin embargo, se observa la falta de relatos en la literatura sobre los diferentes padrones morfométricos del acromion. El objetivo de este estudio fue establecer un padrón morfométrico del acromion, a través de la determinación de su largo, grosor y proyección anterior, además de determinar su relación con el proceso coracoides y el tubérculo supraglenoideo. Fueron analizadas 60 escápulas (37 derechas y 23 izquierdas) de individuos adultos, de la colección de piezas óseas del Departamento de Anatomía de la Universidad Federal de Pernambuco, Brasil. Con la ayuda de un paquímetro y una cinta métrica de perimetría fueron realizadas medidas del largo, grosor y proyección anterior del acromion y también de las distancias del acromion al tubérculo supraglenoideo y al proceso coracoides. Los resultados obtenidos fueron: largo del acromion: 4,96cm $\pm 0,46(74 \%)$; grosor: $0,72 \mathrm{~cm} \pm 0,1$ (72\%); proyección anterior: 4,11cm \pm $0,42(67 \%)$; distancia entre el acromion y el tubérculo supraglenoideo: $2,95 \mathrm{~cm} \pm 0,35(67 \%)$; distancia entre el acromion y el proceso coracoides: $3,8 \mathrm{~cm} \pm 0,52(64 \%)$. No observamos variaciones entre las escápulas derechas e izquierdas. El análisis morfométrico del acromion debe ser utilizado como base auxiliar para los estudios, procurando un mejor conocimiento de las patologías que existen en esa región.

PALABRAS CLAVE: Acromion; Morfometría; Articulación humeral.

\section{INTRODUCCIÓN}

El síndrome del impacto, también conocido como síndrome del pinzamiento del espacio subacromial (SPES), es definido como un síndrome doloroso localizado en los tendones del manguito rotador del hombro en el compartimiento subacromial demarcado por el arco coracoacromial y la cabeza humeral por abajo (Greve et al., 1992). Este síndrome es clínicamente caracterizado por el dolor del hombro debido a mecanismos primarios y secundarios de la irritación del manguito rotador (Mayerhofer \& Breitenseher, 2004). Los factores primarios son la curvatura y el aspecto anterior y ganchoso del acromion, y los factores secundarios son principalmente la tendinitis calcárea, inestabilidad glenohumeral y procesos articulares degenerativos relacionados a la articulación acromioclavicular (Mayerhofer \& Breitenseher). Neer (1972) describió la condición clínica de este síndrome como la producción del dolor en la región lateral del múscu- lo deltoides cuando el miembro es levantado y la escápula estabilizada.

Estudios referentes a las causas de las patologías del manguito rotador, relatan que están relacionadas con enfermedades degenerativas de los tendones musculares o a mecanismos extrínsecos de la compresión debido a acromion ganchosos y del arco coracoacromial (Gill et al., 2002). De acuerdo con Neer (1983), 95\% de estas afecciones son causadas por el impacto.

El SPES fue considerado por muchos autores como un importante factor causante de las lesiones del manguito rotador, y puede deberse a que el acromion en su cara inferior presenta una prominente inclinación que modifica las dimensiones del espacio subacromial (Nicoletti et al., 1990).

\footnotetext{
* Alumno del curso de Fisioterapia de la Universidad Federal de Pernambuco, Brasil.

** Fisioterapeuta, Universidad Federal de Pernambuco, Brasil.

${ }^{* * *}$ Prof. Dra. del Departamento de Anatomía de la Universidad Federal de Pernambuco, Brasil.
} 
Estas alteraciones que llevan a lesiones del manguito rotador pueden también estar relacionadas con la presencia de osteofitos (Chambler et al., 2003; Mahakkanukrauh \& Surin, 2003; Pearsall et al., 2003; Youm et al., 2005).

En 1986, Bigliani et al., describieron en cadáveres tres diferentes formas de acromion: tipo I (plano), tipo II (curvo) y tipo III (ganchoso). La forma y la concavidad del acromion pueden estar relacionadas con el desarrollo de la patología en el espacio subacromial acelerando la patología degenerativa en este espacio (Lisy et al., 2004). Estudios demostraron que acromion tipo III es más frecuente en poblaciones que sufren patologías del manguito rotador y síndrome del impacto (Morrison \& Bigliani, 1987; Neer, 1983).

Estudios que relatan la morfología del acromion y sus relaciones con el tubérculo supraglenoídeo y el proceso coracoides son de fundamental importancia en la determinación de la dimensión del espacio subacromial, teniendo en cuenta que las variaciones de estas estructuras pueden provocar el principio de SPES. Debido a la falta de datos en la literatura sobre los diferentes padrones morfométricos del acromion, este estudio fue llevado a cabo con el objetivo de establecer dicho padrón morfométrico, por medio de la determinación de su largo, grosor y proyección anterior, y además, establecer su relación con el proceso coracoides y el tubérculo supraglenoídeo.

\section{MATERIAL Y MÉTODO}

Fueron analizadas 60 escápulas ( 37 derechas y 23 izquierdas) de individuos adultos de la colección de piezas óseas del Departamento de Anatomía de la Universidade Federal de Pernambuco, Brasil. No fueron considerados aspectos, raza, edad y sexo. Las medidas fueron tomadas con la ayuda de un paquímetro (marca Vernier, precisión de $0,02 \mathrm{~mm}$ ) y una cinta métrica de perimetría.

El largo del acromion fue tomado con la cinta métrica, localizada en la cara superior de la estructura ósea, a partir del punto más anterior de su extremidad libre hasta su base, en la unión con la espina escapular (Fig.1). El grosor fue tomado con el paquímetro, localizado verticalmente, con el mínimo de inclinación posible y perpendicular al acromion, en un punto preestablecido con la cinta métrica referente al tercio de su longitud, a partir de su extremidad libre (Fig.2). La proyección anterior fue obtenida con el paquímetro posicionado paralelo a la cara superior del acromion por medio de los puntos prominentes de sus extremidades anterior y posterior (Fig. 3). La distancia entre el acromion y el proceso coracoides (distancia A-PC)fue establecida con la ayuda del paquímetro a partir de las extremidades libres del proceso coracoides y del acromion (Fig. 4). La medida de la distancia del acromion al tubérculo supraglenoideo (distancia A-TSG) fue realizada con el paquímetro posicionado en la superficie inferior de la extremidad del acromion hasta el punto más superior del tubérculo supraglenoideo.

Las medidas fueron evaluadas en relación a la lateralidad y fueron aplicados el test $t$ de Student para las variaciones del largo y proyección anterior del acromion, distancia entre éste y el tubérculo supraglenoideo, y el test Mann-Whitney para las variaciones del ancho del acromion y la distancia de éste al proceso coracoides. La significación estadística fue considerada admisible a un nivel crítico de $5 \%$ en todos los casos.

\section{RESULTADOS}

Los valores medios y porcentuales encontrados para el largo, grosor y proyección anterior del acromion fueron, respectivamente: 4,96 $\pm 0,46$ (74\%); 0,72 $\pm 0,1$ (72\%); $4,11 \pm 0,42(67 \%)$. Las distancias del acromion al tubérculo supraglenoideo y al proceso coracoides presentaron, respectivamente, los siguientes valores medios: $2,95 \pm 0,35$ (67\%); 3,8 $\pm 0,52$ (64\%). Los valores medios de las variaciones referentes a las escápulas derechas e izquierdas se expresan en la Tabla I. No fue observada diferencia cuando se compararon las escápulas izquierdas y derechas.

Tabla I. Valores medios de las escápulas derechas e izquierdas de individuos adultos de la colección de piezas óseas del Departamento de Anatomía de la Universidade Federal de Pernambuco, Brasil.

\begin{tabular}{lcccc}
\hline & Escápulas derechas & \% & Escápulas Izquierdas & $\%$ \\
Longitud & $4,93 \pm 0,47$ & 78 & $5,12 \pm 0,46$ & 65 \\
Grosor & $0,72 \pm 0,12$ & 73 & $0,71 \pm 0,06$ & 82 \\
Proyección anterior & $4,09 \pm 0,43$ & 62 & $4,14 \pm 0,4$ & 65 \\
Distancia A-TSG & $2,99 \pm 0,38$ & 75 & $2,94 \pm 0,34$ & 78 \\
Distancia A-PC & $3,79 \pm 0,49$ & 59 & $3,83 \pm 0,56$ & 65 \\
\hline
\end{tabular}

Valores en cm; resultados expresados como media \pm desviación estándar.Test t de student y test de Mann Whitney; p<0,05. 


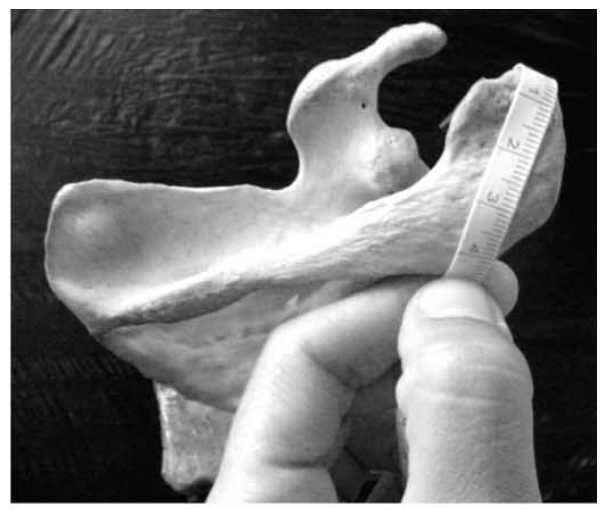

Fig. 1. Longitud del acromion.

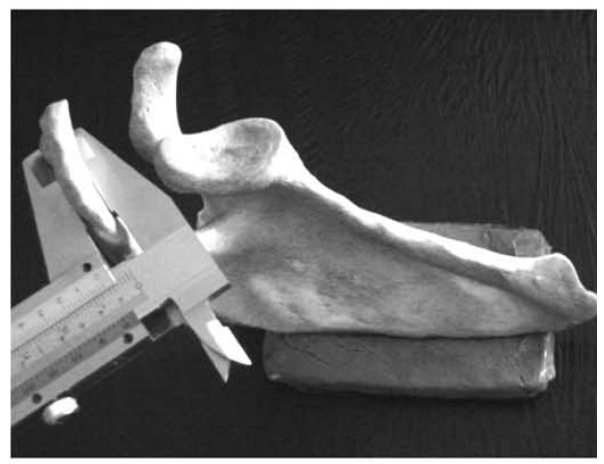

Fig. 2. Grosor del acromion.

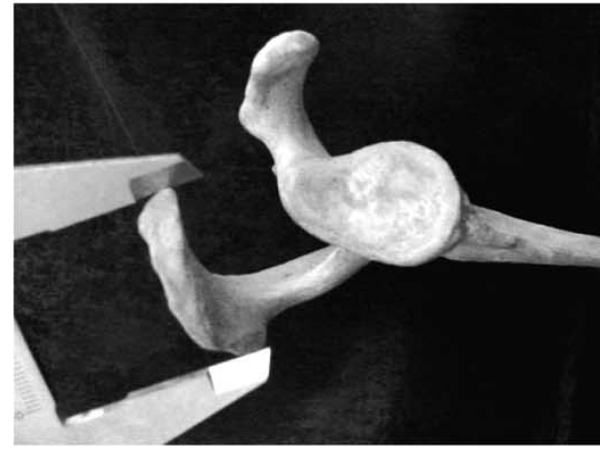

Fig. 3. Proyección anterior del acromion.

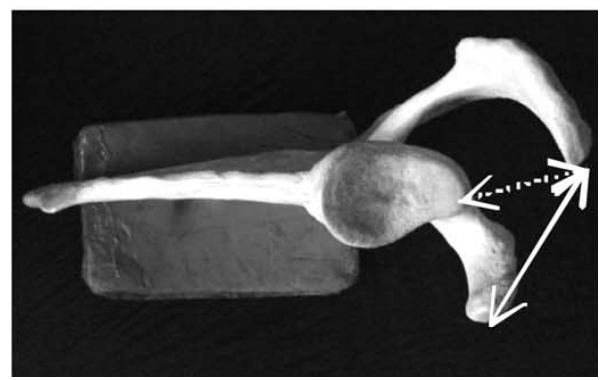

Fig. 4. Distancia entre el acromion y el proceso coracoides (flecha continua) y distancia entre el acromion y el tubérculo supraglenoideo (flecha punteada).

\section{DISCUSIÓN}

Con respecto a la evaluación morfométrica del largo, grosor y proyección anterior del acromion, así como, las distancias del acromion al tubérculo supraglenoideo y al proceso coracoides, no fueron observadas diferencias de los parámetros morfométricos de las escápulas drechas e izquierdas, confirmando los hallazgos de von Schroeder et al. (2001).

Nicoletti et al. correlacionaron las lesiones del manguito rotador con el grado de inclinación del acromion, demostrando que el lado en que estos procesos dolorosos se instalaron estaba relacionado con el ángulo de inclinación acromial y no con lo carácter diestro o zurdo de los individuos.

En relación a la proyección anterior y al grosor del acromion, observamos valores inferiores a los resultados encontrados por von Schroeder et al. quienes observaron valores medios para la proyección anterior de 4,8 cm $\pm 0,5$ y $0,9 \mathrm{~cm} \pm 0,1$ para el grosor de esta estructura ósea. Estos mismos autores encontraron valores inferiores $(1,6 \mathrm{~cm} \pm 0,2)$ referentes a la distancia entre el acromion y el tubérculo supraglenoídeo en relación a nuestros hallados. Del mismo modo, la distancia entre el acromion y el proceso coracoides encontrado en nuestro estudio fue inferior a los hallados von Schroeder et al. $(2,7 \mathrm{~cm} \pm$ $0,5)$. Es importante destacar las diferencias entre las metodologías utilizadas para las tomar las medidas entre los dos estudios.

Entre las posibles causas del pinzamiento subacromial están las variaciones morfológicas del acromion (Nicoletti \& Manso, 1995; Greve et al., 1995). A pesar de haber evaluado la curvatura del acromion, es importante destacar la falta de datos sobre este parámetro óseo.

Estudios anatómicos y radiográficos realizados en cadáveres y pacientes portadores de SPES, demostraron variaciones de la curvatura de la cara inferior del acromion, que por existir en el plano sagital, alteraron las dimensiones del espacio encontrado bajo el arco coracoacromial, por donde pasan los tendones del manguito rotador (Nicolletti et al.; Neer).

El acromion muy curvo es considerado importante en la producción de atrición subacromial y en lesiones del manguito rotador (Nicolleti \& Manso). Morrison \& Bigliani analizaron 20 radiografías y encontraron, en $80 \%$ de sus pacientes portadores SPES, acromion muy curvos, creyendo en la correlación entre la ruptura del manguito y acromion con grandes curvaturas. Hirano et al. (2002)sostienen que la presencia de acromion Tipo III no necesariamente debe estar relacionada a la lesión del manguito rotador, sin embargo, influencia la gravedad de esta afección. El estudio de Prato et al. (1998) investigó el ángulo de inclinación del acromion, el cual fue inferior en el grupo con patologías del manguito rotador cuando fue comparado con otro grupo que no presentaba lesión en esta estructura. Esto último sugiere que, cuanto menor es la medida del ángulo de inclinación estudiado por Prato et al., menor será la distancia entre el acromion y el tubérculo supraglenoídeo.

Así, datos referentes al padrón morfométrico del acromion podrán servir de base para futuros estudios, promoviendo un mejor conocimiento de patologías que pueden estar influenciadas por estas variaciones morfológicas. 
TORRES, C. A.; RIBEIRO, C. A. S.; MAUX, S. X. D. A.; OLIVEIRA, G. C. D.; NEVES, M. G.; SALGADO, A. R. F.; ANDRADE, F. R.; BRITO, C. V. \& MORAES, A. S. R. Morphometry of acromion and its clinical implications. Int. J. Morphol., 25(1):51-54, 2007.

SUMMARY: Previous studies demonstrated that the morphology of the acromion and its respective relations with the uppermost point of the glenoid and the coracoid process is very important in the determination of subacromial space. The variations of these structures can predispose the beginning of the Impingement Syndrome. However, there is a shortage of reports on literature about the different acromion's morphometric samples. The purpose of this study was to determine a morphologic pattern of the acromion, through the determination of its length, thickness, front projection, and its relation with the uppermost point of the glenoid and the coracoid process. We used 60 scapulae (37 right and 23 left) of adults from Departamento de Anatomia da Universidade Federal de Pernambuco, Brazil. To measure the length, thickness and front projection of acromion, and the distances from acromion to the uppermost point of the glenoid and to the coracoid process were used a calipers and a tape measure. We found these results: acromion length: $4.96 \mathrm{~cm} \pm 0.46$ (74\%); thickness: $0.72 \mathrm{~cm} \pm 0.1$ (72\%); front projection: $4.11 \mathrm{~cm} \pm 0.42(67 \%)$; distance between acromion and the uppermost point of the glenoid: $2.95 \mathrm{~cm} \pm 0.35$ (67\%); distance between acromion and coracoid process: $3.8 \mathrm{~cm} \pm 0.52(64 \%)$. We did not find differences between right and left scapulaes. The morphometric analysis of the acromion should be used like an auxiliary basis to studies that promote a better knowledge about the disease that appear in this area.

KEY WORDS: Acromion; Morphometry; Shoulder joint.

\section{REFERENCIAS BIBLIOGRÁFICAS}

Bigliani, L. U.; Morrison, D. S. \& April, E.W. The morphology of the acromion and its relationship to rotator cuff tears. Orthop. Trans., 10:228,1986.

Chambler, A. F. W.; Pitsillides, A. A. \& Emery, R. J. H. Acromial spur formation in patients with rotator cuff tears. J. Shoulder Elbow Surg., 12(4):314-321, 2003.

Gill, T. J. M. D.; McIrvin, E. B. S.; Kocher, M. S. M. D.; Homa, K. M. S.; Mair, S. D. M. D. \& Hawkins, R. J. M. D. The relative importance of acromial morphology and age respect to rotator cuff pathology. J. Shoulder Elbow Surg., 11:327-30, 2002.

Greve, J. M. D’A.; Ferreira Filho, A. A.; Zoppi Filho, A.; Bolliger Neto, R. \& Yoshinari, N. H. Síndrome do impacto: Anatomia e biomecânica do ombro e correlações clínicas com a Síndrome do Impacto. Bras. Reumatol., 32:89-94, 1992.

Greve, J. M. D’A.; Ferreira Filho, A. A.; Zoppi Filho, A. \& Neto, R. B. Síndrome do Impacto - Relações Anátomo-clínicas, Diagnóstico, Tratamento. Medicina de Reabilitação, 41:17-24, 1995.

Hirano, M.; Ide, J. \& Takagi, K. Acromial shapes and extension of rotator cuff tears: Magnetic resonance imaging evaluation. $J$. Shoulder Elbow Surg., 11:576-8, 2002.

Lisy, M.; Hreusik, P. \& Steno, B. The shape of the acromion and its effect on the subacromial space. Acta Chir. Orthop. Traumatol. Cech., 71(2):110-4, 2004.

Mahakkanukrauh, P. \& Surin, P. Prevalence of osteophytes associated with the acromion and acromioclavicular joint. Clin. Anat., 16(6): 506-10, 2003.

Mayerhofer, M.E. \& Breitenseher, M.J. Impingment syndrome of the shoulder. Radiologe, 44(6): 569-77, 2004.

Morrison, D. S. \& Bigliani, L. U. The clinical significance of variations in acromial morphology. Orthop. Trans., 11:234, 1987.
Neer, C. S. II. Anterior acromioplasty for the chronic impingement syndrome of the shoulder. A preliminary report. J. Bone Joint Surg., 54:41-50, 1972.

Neer, C. S. II. Impingement lesions. Clin. Orthop., 173:70-7, 1983.

Nicoletti, S. J.; Filho, J.L. \& Faloppa, F. Avaliação radiográfica do ângulo de inclinação do acrômio em indivíduos normais. Bras. Ortop., 25:161-4, 1990.

Nicoletti, S. \& Manso, G. Artroscopia do ombro: reavaliando o papel da morfologia do acrômio na produção do pinçamento subacromial. Bras. Ortop., 30:674-8, 1995.

Pearsall, A.W.; Bonsell, S.; Heitman, R. J.; Helms, C. A.; Osbahr, D. \& Speer, K.P. Radiographic findings associated with symptomatic rotator cuff tears. J. Shouder Elbow Surg., 12:1227, 2003.

Prato, N.; Peloso, D.; Franconeri, A.; Tegaldo, G.; Ravera, G. B.; Silvestri, E. \& Derchi, L. E. The anterior tilt of the acromion: radiographic evaluation and correlation with shoulder diseases. Eur. Radiol., 8:1639-46, 1998.

von Schroeder, H. P.; Kuiper, S. D. \& Botte, M. J. Osseous anatomy of the scapula. Clin. Orthop. Rel. Res., 383:131-9, 2001.

Youm, T.; Hommen, J. P.; Ong, B. C.; Chen, A. L. \& Shin, C. Os acromiales: evaluation and treatment. Am. J. Orthop., 34(6):277-83, 2005.

Dirección para correspondencias:

Dra. Sílvia Regina Arruda de Moraes.

Rua Capitão Rui Lucena, 160/501

CEP: $50070-080$

Boa Vista - Recife - PE

BRASIL

Email: sramoraes@gmail.com

Recibido : 04-09-2006

Aceptado: 22-12-2006 